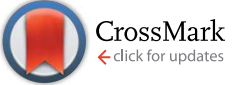

Cite this: RSC Adv., 2017, 7, 2180

Received 15th October 2016 Accepted 28th November 2016

DOI: $10.1039 / \mathrm{c} 6 \mathrm{ra} 25290 \mathrm{~g}$

www.rsc.org/advances

\title{
Construction and functional assessment of zein thin film incorporating spindle-like $\mathrm{ZnO}$ crystals
}

\author{
Ying Cao, ${ }^{a}$ Ting-Ting Chen, ${ }^{a}$ Wei Wang, ${ }^{\mathrm{b}}$ Meng Chen ${ }^{\mathrm{a}}$ and Hua-Jie Wang ${ }^{\text {*ac }}$
}

The use of plastics has rapidly progressed during recent years in food packaging, which can be attributed to technological innovations in the synthesis of plastics and lower costs. However, the massive accumulation of plastic waste is often accompanied with their excessive consumption because of their poor degradability. Consequently, interest in the native materials is growing. It is noted that zein is a corn protein, has good compatibility and has been widely applied in the food industry. However, microbial contamination is a huge detriment to its long-term applications. This study aims to identify a method, which can provide antibacterial activity to a zein thin film. Monodispersed and spindle-like ZnO crystals made of nanocrystals were synthesized and added into zein thin films. EDX mapping confirmed the homogeneous distribution of $\mathrm{ZnO}$ in films. The antibacterial activities have been approved and showed great stability. In summary, the potential of zein thin films incorporating $\mathrm{ZnO}$ crystals as a functional packaging film has been demonstrated.

\section{Introduction}

Food-borne diseases are becoming one of the serious problems faced by humans along with environmental contamination. ${ }^{1}$ Technological innovations in food safety related to consumer confidence and human health are becoming extremely urgent. Traditional synthetic plastics have been widely applied in food packaging in an accelerated mode. Although they are from lowcost raw materials and have good mechanical properties, poor biodegradability is one of their fatal flaws and it is resulting in a massive accumulation of plastic waste and subsequent serious environmental problems. ${ }^{2}$ Therefore, scientists are concentrating their efforts on finding new techniques and packaging materials. ${ }^{3}$

Biodegradable and natural polymers have recently aroused great attention for their relative abundance, film-forming ability or nutritional qualities. ${ }^{4,5}$ Especially, the appearance of slowrelease technique drives the development of the native polymers-based active packaging, which is becoming a highlight in food industry. ${ }^{6-8}$ For example, Dalarbashi et al. fabricated a new active packaging film made from a soluble soybean polysaccharide incorporating $\mathrm{ZnO}$ nanoparticles,

\footnotetext{
${ }^{a}$ Collaborative Innovation Center of Henan Province for Green Manufacturing of Fine Chemicals, Key Laboratory of Green Chemical Media and Reactions, Ministry of Education, School of Chemistry and Chemical Engineering, Henan Normal University, Xinxiang, Henan 453007, P. R. China. E-mail: wanghuajie972001@163. com; Fax: +86-373-3328507; Tel: +86-373-3326335

${ }^{b}$ Pharmaceutical College, Henan University, Kaifeng, Henan 475004, P. R. China ${ }^{c}$ Center for Advanced Materials Research, Zhongyuan University of Technology, No. 1 Huaihe Road, Xinzheng Shuanghu Economic Development Zone, Zhengzhou 451191, P. R. China
}

which showed good antibacterial, antifungal and yeasticidal activities. ${ }^{9}$

This study aimed to prepare an active packaging made of zein and ZnO crystals. Herein, biomimetic synthesis, characterizing with environmental-friend and facile conditions, has been used to fabricate spindle-like $\mathrm{ZnO}$ crystals. Zein, a predominant storage protein of corn, has exhibited great biodegradability and excellent biocompatibility, and can act as the structure-directing agent for nanocrystal synthesis and the formation of films. ${ }^{10-17}$ EDX mapping confirmed that $\mathrm{ZnO}$ crystals could homogeneously distribute in zein thin films. The zein thin film incorporating ZnO crystals could effectively inhibit the growth of Escherichia coli (E. coli) and Staphylococcus aureus (S. aureus), and these antibacterial activities could maintain a long-term effect. All of these results support the conclusion that zein thin films incorporating ZnO crystals are suitable to act as a functional packaging materials.

\section{Material and methods}

\subsection{Materials}

Zein was purchased from Sigma (St. Louis, MO, USA). Zinc chloride $\left(>99.999 \%, \mathrm{MW}=136.3 \mathrm{~g} \mathrm{~mol}^{-1}, \mathrm{AR}\right)$ was purchased from Tianjin Chemical Reagent Factory (Tianjin, China). All other solvents and chemicals were analytical grade.

\subsection{Biomimetic synthesis of spindle-like zein-conjugated ZnO crystals with hierarchical structure}

Spindle-like zein-conjugated ZnO crystals with a hierarchical structure were prepared by a modified biomimetic method using zein as the structure-directing agent. Briefly, $20 \mathrm{~mL}$ of 
a $6 \mathrm{mg} \mathrm{mL^{-1 }}$ zinc chloride aqueous solution was quickly added dropwise into $40 \mathrm{~mL}$ of a $2 \mathrm{mg} \mathrm{mL}^{-1}$ zein alcoholic aqueous solution $(60 \%)$ under stirring. The $\mathrm{pH}$ value of the mixture was adjusted to 8.0-8.9 using a $1 \mathrm{M} \mathrm{NaOH}$ solution and the mixture was kept static for $30 \mathrm{~min}$ at $37^{\circ} \mathrm{C}$. Finally, the as-prepared sample was separated by centrifugation for $10 \mathrm{~min}$ at $10000 \mathrm{rpm}$. The collected product was washed with distilled water and lyophilized under vacuum for $48 \mathrm{~h}$.

\subsection{Fabrication of zein thin films incorporating $\mathrm{ZnO}$ crystals}

Zein (600 mg) and zein-conjugated ZnO crystals (100 mg) were completely dispersed into $75 \%$ alcoholic aqueous solution. Then, $3 \mathrm{mg}$ of cysteine, $100 \mu \mathrm{L}$ L-glycerol and $100 \mu \mathrm{L}$ of polyethylene glycol were added in sequence and stirred for $35 \mathrm{~min}$ at $80^{\circ} \mathrm{C}$. Following this, the films were cast by pouring the mixture onto polystyrene Petri dishes and fumigated for $2 \mathrm{~h}$ at $70{ }^{\circ} \mathrm{C}$ in a water bath. The thin films were peeled from the casting surface and stored in a humidified incubator.

\subsection{Characterization}

The surface structure and morphology of the zein-conjugated ZnO crystals were observed using a field emission scanning electron microscope (Merin Compact, Zeiss, German) and the distribution of ZnO in the zein thin films was detected by EDX mapping. Furthermore, the inner structure of the $\mathrm{ZnO}$ crystals was observed using a JEM-2010 electron microscope working at $200 \mathrm{kV}$.

Fourier transform infrared spectroscopy (FTIR) was applied to confirm the organic composition of the zein-conjugated $\mathrm{ZnO}$ crystals. The analysis was carried out on a Bio-Rad FTS-40 Fourier transform infrared spectrophotometer in the wavenumber range of $4000-400 \mathrm{~cm}^{-1}$. The spectra were collected at a $2 \mathrm{~cm}^{-1}$ resolution with 128 scans by preparing $\mathrm{KBr}$ pellets with a $3: 100$ "sample-to-KBr" ratio. Moreover, the organic content in the ZnO crystals was quantified by thermogravimetricdifferential scanning calorimetry (TG/DTA) on an EXSTAR TG/ DTA 6300 instrument (Seiko, Japan).

To confirm the composition of the inorganic component, XRD measurements were performed on a Bruker D8 \& Advance X-ray powder diffractometer with graphite monochromatized $\mathrm{Cu} / \mathrm{K} \alpha(\gamma=0.15406 \mathrm{~nm})$. A scan rate of $0.05 \mathrm{deg} \mathrm{s}^{-1}$ was applied to record the pattern in the $2 \theta$ range of $10-80^{\circ}$.

The stability of the thin films was determined in an aqueous solution with different $\mathrm{pH}$ values. Briefly, the thin films were immersed into an aqueous solution at different time points and the $\mathrm{Zn}$ ion content in solution was quantified by atomic absorption spectrometry (AAS).

\subsection{Enzymolysis}

The zein thin film (diameter $=15 \mathrm{~mm}, n=3$ ) was immersed into $4 \mathrm{~mL}$ of an enzyme solution containing $12.5 \mathrm{U} \mathrm{mL}^{-1}$ collagenase and incubated at $37{ }^{\circ} \mathrm{C}$ under static conditions. Each time the enzyme solution with the same volume as that taken out was added to the solution. The degradation rate was analysed spectrophotometrically according to our previous study. ${ }^{14}$

\subsection{Water vapour permeability (WVP)}

The WVP of the zein thin film was determined according to the report of Abdollahi et al. ${ }^{18}$ Briefly, glass permeation cups (diameter $=30 \mathrm{~mm}, \mathrm{n}=3$ ) containing distilled water were covered with the zein thin film and kept static at $20^{\circ} \mathrm{C}$ and $1.5 \%$ relative humidity. The weight of the cup was recorded at $1 \mathrm{~h}$ intervals for $8 \mathrm{~h}$. The WVP was calculated as follows:

$$
\mathrm{WVP}=\mathrm{WVTR} \times L / \Delta P
$$

where WVTR is the measured water vapour transmission rate ( $\mathrm{g}$ $\mathrm{m}^{-2} \mathrm{~s}^{-1}$ ) through the film, $L$ is the mean film thickness (m), and $\Delta P$ is the partial water vapour pressure difference $(\mathrm{Pa})$ across the two sides of the film.

\subsection{Antibacterial activity}

The antibacterial activities of the thin films were evaluated using the disc diffusion method and two microorganism strains of $E$. coli (Gram-negative bacteria) and $S$. aureus (Gram-positive bacteria) were chosen. Briefly, all microorganism pre-inoculum cultures were grown overnight at $37{ }^{\circ} \mathrm{C}$ in $20 \mathrm{~mL}$ of nutrient broth (made of $1 \mathrm{~g} \mathrm{~L}^{-1}$ beef extract; $5 \mathrm{~g} \mathrm{~L}^{-1}$ neutralized peptone; $2 \mathrm{~g} \mathrm{~L}^{-1}$ yeast extract; $5 \mathrm{~g} \mathrm{~L}^{-1} \mathrm{NaCl}$ ) and subjected to horizontal shaking at $100 \mathrm{rpm}$. The samples (diameter $=6 \mathrm{~mm}, n=6$ ) were placed onto the nutrient agar that was previously inoculated with different microorganisms. After incubation for $24 \mathrm{~h}$, the presence of a zone of growth inhibition below and around the specimen was checked by visual inspection.

\subsection{Statistics}

All data represented as the mean $\pm \mathrm{SD}$ and the number of the independent replicates is individually shown for each experiment. The analysis of data was carried out by one-way factorial analysis of variances (ANOVA) and multiple comparisons (Fisher's method as post hoc test, $p<0.05$ ).

\section{Results and discussion}

The specific requirements for environment protection and food safety drive the innovation of food packaging with an emphasis on the roles of biodegradability and antibacterial properties. ${ }^{19}$ This study focused on understanding the feasibility to construct a zein thin film incorporating ZnO crystals. We firstly developed a modified biomimetic synthesis route to fabricate zein conjugated ZnO crystals. This route had two advantages. First, the entire synthesis route could be accomplished under ecofriendly and gentle conditions, which will reduce the dubious factors from an organic system and investment cost from special equipment. Second, the participation of zein in spindlelike $\mathrm{ZnO}$ crystals will be helpful for their better distribution in a zein thin film. With our interest in active food packaging, we envisaged that a zein thin film incorporating ZnO crystals will generate a new class of active food packaging that could be biodegradable and antibacterial.

The XRD analysis clearly demonstrates the formation of $\mathrm{ZnO}$ crystals as shown in Fig. 1A. It can be seen that eight 


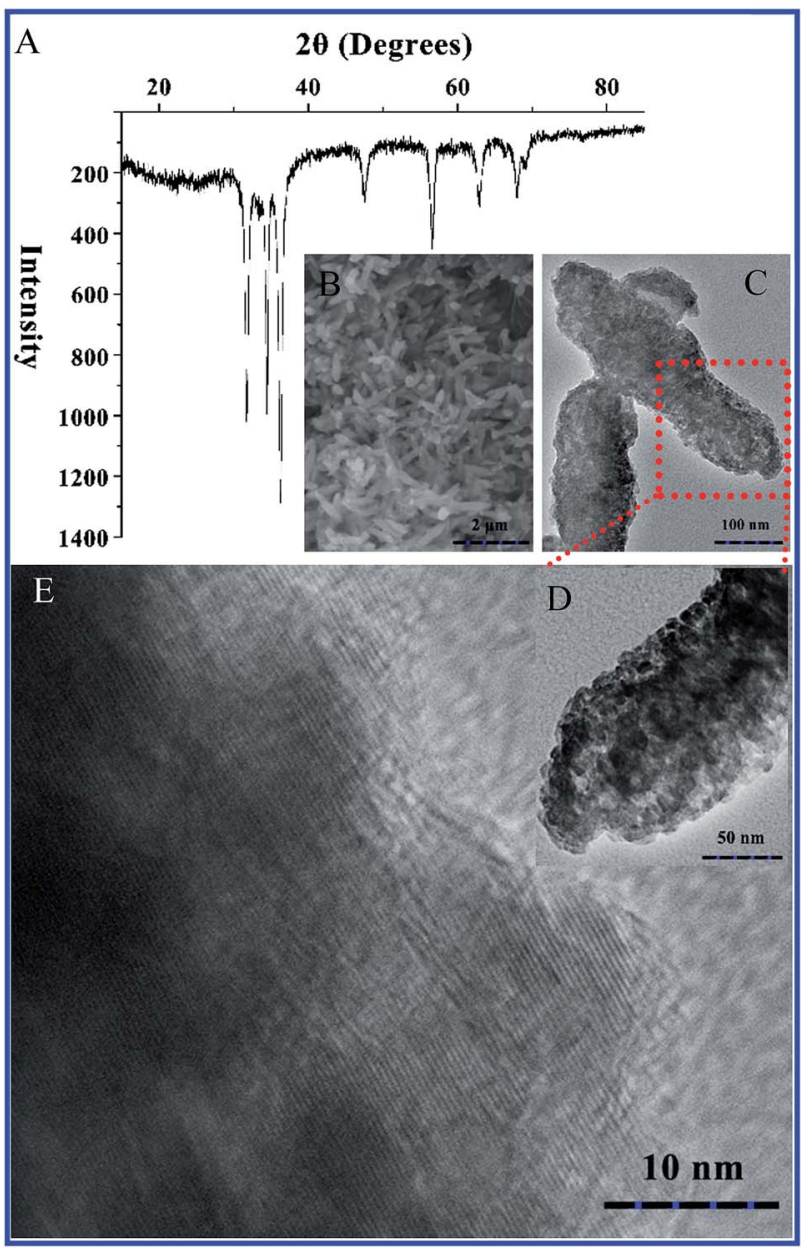

Fig. 1 Analysis on the inorganic component and morphologies of zein conjugated ZnO crystals. (A) XRD spectrum; (B) SEM observation; (CE) TEM observation.

characteristic peaks for $\mathrm{ZnO}$ appear at $2 \theta$ at $31.769^{\circ}, 34.421^{\circ}$, $36.252^{\circ}, 47.538^{\circ}, 56.602^{\circ}, 62.862^{\circ}, 66.378^{\circ}, 67.961^{\circ}$ and $69.098^{\circ}$, corresponding to $\mathrm{ZnO}$ (100), (002), (101), (102), (110), (103), (200), (110) and (201) diffractions. These peaks are in good agreement with the reported data of the JCPDS card 36-1451. In this study, we supplemented $60 \%$ ethanol into the reactive system instead of high temperature to promote the transformation from $\mathrm{Zn}(\mathrm{OH})_{2}$ to $\mathrm{ZnO}^{20}$ Moreover, biomimetic synthesis allows us to control the morphologies and size of nanocomposites using native molecules as templates. ${ }^{21,22}$ Both SEM and TEM clearly confirm that zein acts as a good transport carrier in Ostwald ripening and induces the formation of a spindle-like structure that is comprised of nanoparticles (Fig. 1B-E). ${ }^{23}$

Fig. 2 shows the FTIR spectrum of pure ZnO crystals, pure zein and zein conjugated $\mathrm{ZnO}$ crystals. It can be seen that the typical $\mathrm{Zn}-\mathrm{O}$ stretching band in pure $\mathrm{ZnO}$ crystals appears between $430 \mathrm{~cm}^{-1}$ and $520 \mathrm{~cm}^{-1}$ (Fig. 2A). ${ }^{24-26}$ As for pure zein, the peaks at $3396 \mathrm{~cm}^{-1}, 2958 \mathrm{~cm}^{-1}, 1651 \mathrm{~cm}^{-1}$ and $1539 \mathrm{~cm}^{-1}$ are clearly separated and can be assigned to the stretching vibration of a hydroxyl group, amide $\mathrm{A}^{\prime}$, and amide I and amide

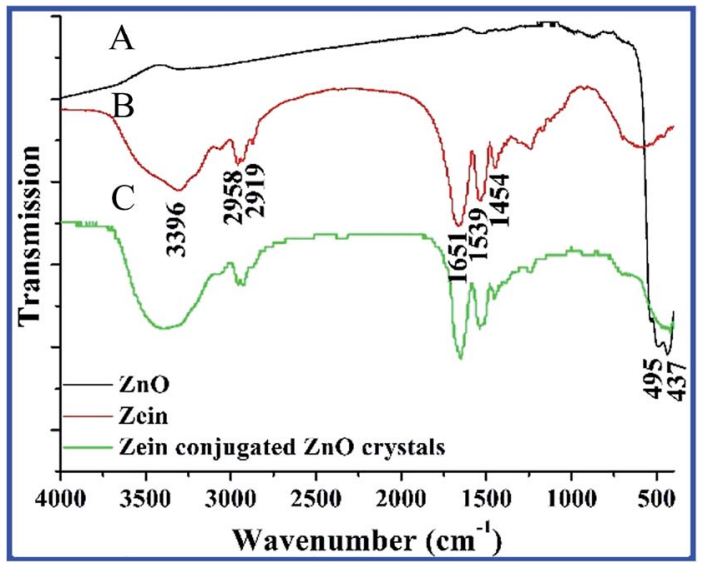

Fig. 2 Analysis on the organic component of zein conjugated $\mathrm{ZnO}$ crystals by FTIR spectra. (A) Pure ZnO, (B) pure zein, and (C) zein conjugated $\mathrm{ZnO}$ crystals.

II, which are the typical protein absorption peaks (Fig. 2B). By comparing Fig. 2A and B, the FTIR spectrum of zein conjugated $\mathrm{ZnO}$ crystals supplies direct evidence of zein participating in the formation of spindle-like $\mathrm{ZnO}$ crystals (Fig. 2C).

According to TG/DTA analysis, we could easily quantify the zein content in $\mathrm{ZnO}$ crystals (Fig. 3A). The total weight loss of the $\mathrm{ZnO}$ crystals decreases to $44.42 \%$ from $250{ }^{\circ} \mathrm{C}$ to $650{ }^{\circ} \mathrm{C}$ according to the TG curve. In the DTA curve, there are three exothermic peaks, appearing at $365{ }^{\circ} \mathrm{C}, 485{ }^{\circ} \mathrm{C}$ and $531{ }^{\circ} \mathrm{C}$,

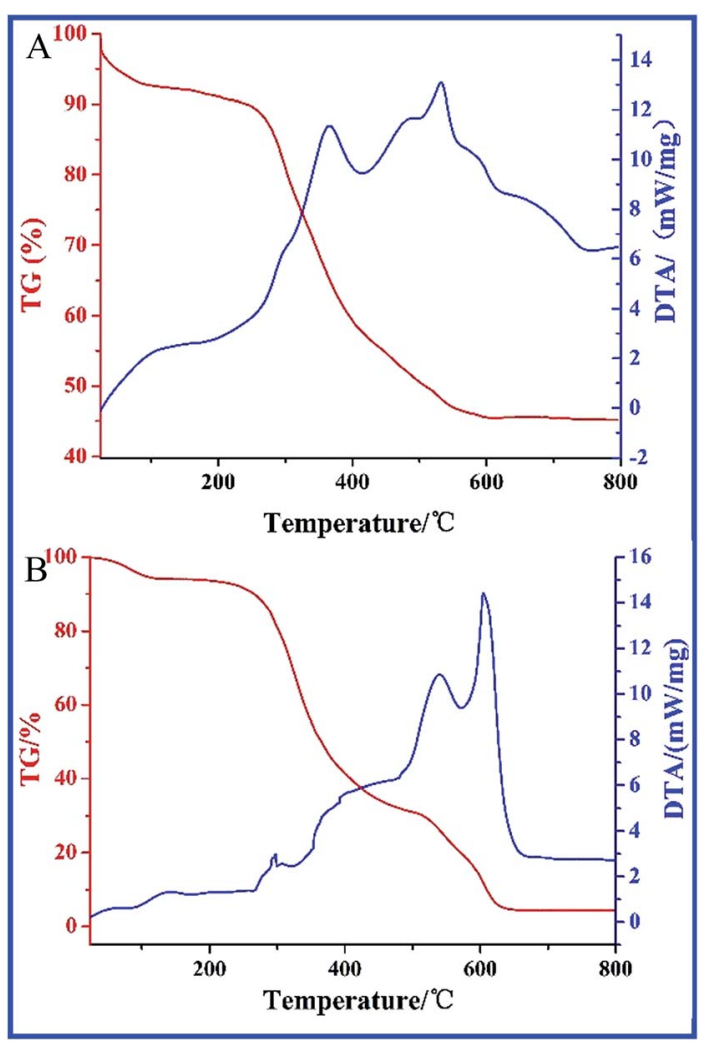

Fig. 3 Analysis on the content of zein in zein conjugated $\mathrm{ZnO}$ crystals by TG/DTA. (A) Zein conjugated ZnO crystals; (B) pure zein. 
respectively, which correspond to the carbonization, side chainburning and main carbon-burning of zein, respectively. ${ }^{27}$ However, by comparison with that of pure zein (Fig. 3B), the peaks of the abovementioned exothermic process appear at $404{ }^{\circ} \mathrm{C}, 538{ }^{\circ} \mathrm{C}$ and $603{ }^{\circ} \mathrm{C}$, respectively, suggesting the stimulative effect of $\mathrm{ZnO}$ on zein burning.

Currently, inorganic nanomaterials in the prevention of bacterial infections are gaining an importance due to low drug resistance and stable physical and chemical properties compared to traditional antimicrobials. Literature studies have demonstrated that $\mathrm{ZnO}$ could effectively inhibit the growth of microorganisms by oxidative stress and the interaction of zinc with the bacteria membrane. ${ }^{\mathbf{2 8 , 2 9}}$ Moreover, these antibacterial activities are species-dependent. ${ }^{30,31}$ For example, Baek and An demonstrated that $S$. aureus was more sensitive than $E$. coli to $\mathrm{ZnO}$ nanoparticles. ${ }^{30}$ Sultana et al. reported that $\mathrm{ZnO}$ nanoparticles exhibited stronger antibacterial activity against $S$. aureus and Bacillus subtilis compared to E. coli and Pseudomonas aeruginosa. $^{31}$ In this study, we also tested the antibacterial activities of zein conjugated $\mathrm{ZnO}$ crystals against $S$. aureus and E. coli (Fig. 4). The results indicate that the $\mathrm{ZnO}$ crystals keep a positive correlation with $\mathrm{ZnO}$ concentrations. At the same time, $S$. aureus shows higher sensitivity than E. coli to $\mathrm{ZnO}$

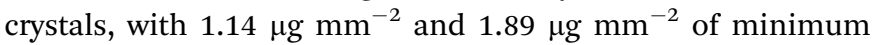
inhibitory concentration, respectively.

Observations of the monodispersed and hierarchical structure and excellent antibacterial activity of zein conjugated $\mathrm{ZnO}$ crystals prompted us to focus on the fabrication of zein thin films incorporating $\mathrm{ZnO}$ crystals. The obtained thin films are yellow and transparent as shown in Fig. 5A. Moreover, we could easily control the thickness by adjusting the content of raw materials. Fig. 5B and $\mathrm{C}$ show the microstructure of the thin film observed by SEM and there are a few remaining air bubbles in the film. As one of the typical elements of a protein, $\mathrm{N}$ could be detected by EDX analysis (Fig. 5D). In addition, we also can detect $\mathrm{Zn}$, which is from $\mathrm{ZnO}$ crystals. By the AAS test, the loading of $\mathrm{ZnO}$ in a thin film can get to $0.66 \mathrm{mg} \mathrm{cm}^{-2}$. The homogeneity of the $\mathrm{ZnO}$ crystals in a thin film was directly observed by EDX mapping based on the distribution of $\mathrm{Zn}$ and N. As shown in Fig. 5E-G, zein has a homogeneous distribution according to $\mathrm{N}$ mapping,

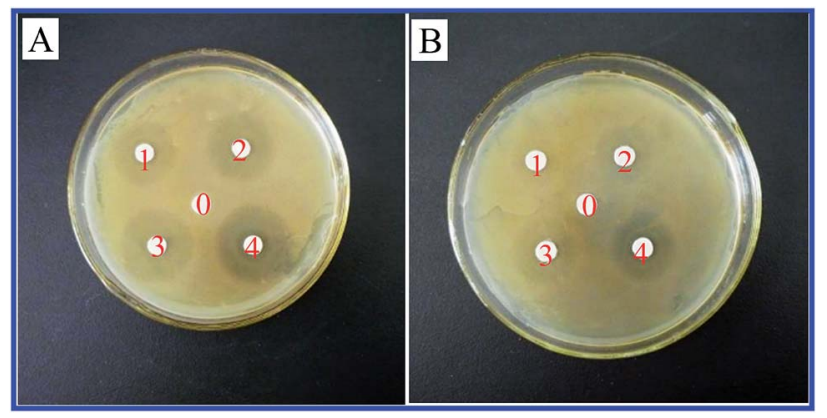

Fig. 4 The antibacterial activity of zein conjugated $\mathrm{ZnO}$ crystals with different concentrations against $S$. aureus (A) and $E$. coli (B) using the disc diffusion method. "0": control; "1": $2.83 \mu \mathrm{g} \mathrm{mm}{ }^{-2}$; "2": $8.49 \mu \mathrm{g}$ $\mathrm{mm}^{-2}$; "3": $14.15 \mu \mathrm{g} \mathrm{mm}^{-2}$; "4": $28.31 \mu \mathrm{g} \mathrm{mm}^{-2}$.

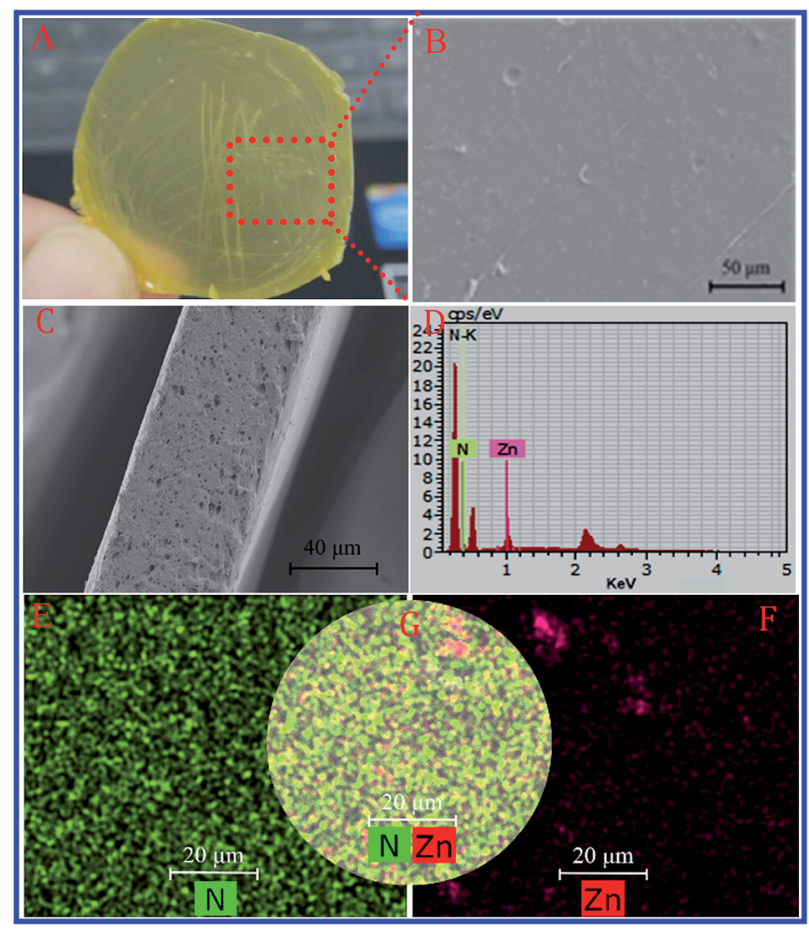

Fig. 5 Morphological observations, elemental composition and phase distribution detection of zein thin films incorporating these crystals. (A) Visual observation; (B) SEM image (facade); (C) SEM image (profile); (D) EDX analysis; (E) EDX mapping on N; (F) EDX mapping on Zn; (G) EDX mapping on $\mathrm{N}$ and $\mathrm{Zn}$.

while the presence of only a slight aggregate of $\mathrm{Zn}$ signals suggests a homogeneous distribution of $\mathrm{ZnO}$ in a thin film.

Protein-based materials are known to undergo naturallycontrolled degradation processes. Zein, the predominant storage protein of corn, has exhibited great biodegradability. In previous studies several groups including our group, have demonstrated that zein could be degraded via enzymolysis. ${ }^{14,15,32}$ In order to understand the biodegradability of a zein thin film, we further studied its degradation process in vitro. A zein thin film $(d=1.5 \mathrm{~cm})$ was immersed into $4 \mathrm{~mL}$ of an enzyme solution containing $12.5 \mathrm{U} \mathrm{mL}^{-1}$ collagenase and incubated at $37{ }^{\circ} \mathrm{C}$ in an incubator under static conditions. As shown in Fig. 6, the degradation rate of the zein thin film increases gradually in 5 days and reaches $66.8 \%$, suggesting its good biodegradability. In addition, we also tested the water vapour permeability of the zein thin film. The result shows that the WVP of the zein thin film incorporating $\mathrm{ZnO}$ crystals reaches $4.15 \pm 0.12\left(\mathrm{~g} \mathrm{~ms}^{-1} \mathrm{~Pa}^{-1}\right) 10^{-11}$. The relatively lower permeability might result from the water-insoluble property of zein. $^{2}$

Active food packaging is currently a focus of attention that is linked to the development of the shelf-life and safety of packaged food. ${ }^{33,34}$ Herein, we investigated the antibacterial activities of a zein thin film incorporating $\mathrm{ZnO}$ crystals against various bacterial pathogens. As shown in Fig. 7, thin films can effectively inhibit the growth of $S$. aureus and E. coli. Furthermore, the inhibition zone size reached $25.39 \pm 0.90 \mathrm{~mm}$ against 


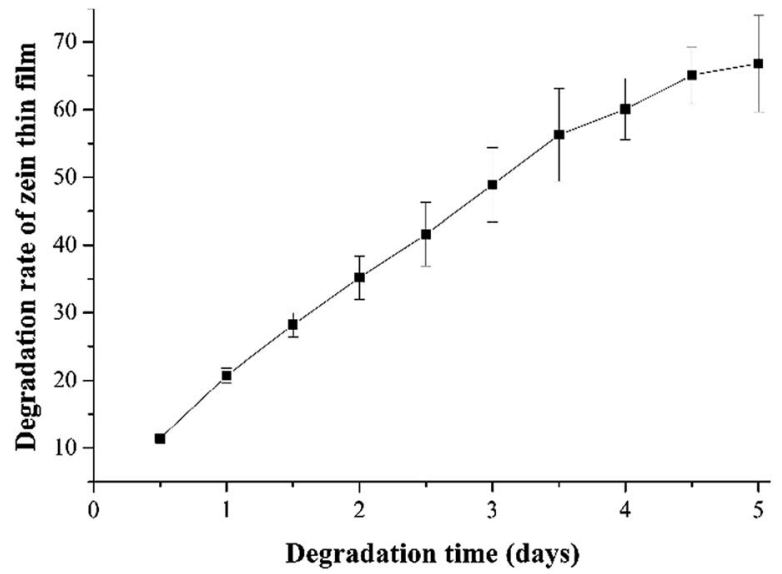

Fig. 6 Degradation of zein thin film by $12.5 \mathrm{U} \mathrm{mL}^{-1}$ collagenase.

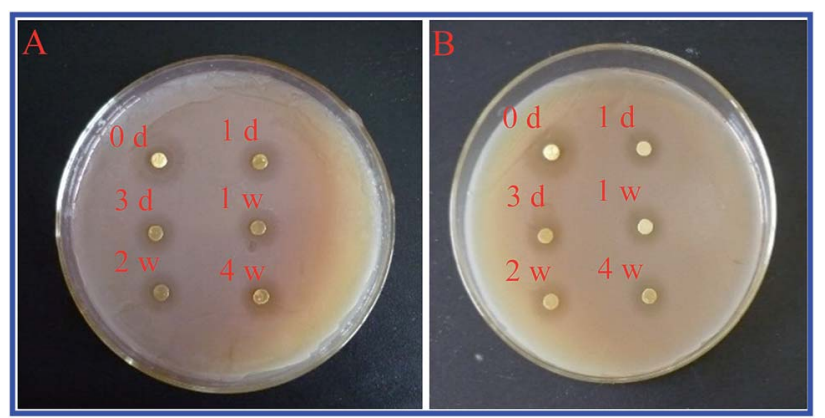

Fig. 7 The antibacterial activity of a zein thin film incorporating $\mathrm{ZnO}$ crystals after immersion in an aqueous solution at different times. (A) $S$. aureus, (B) E. coli.

$S$. aureus and $21.09 \pm 1.89 \mathrm{~mm}$ against $E$. coli. Interestingly, the zein thin films incorporating $\mathrm{ZnO}$ crystals exhibited continuous antibacterial activities. After 4 weeks of immersion in the aqueous solution, the thin film still maintained a high inhibitory effect on both bacterial pathogens.

In order to understand the antibacterial behaviours, we analysed the stability of zein thin films incorporating $\mathrm{ZnO}$ crystals in an aqueous solution with different $\mathrm{pH}$ values (Fig. 8).

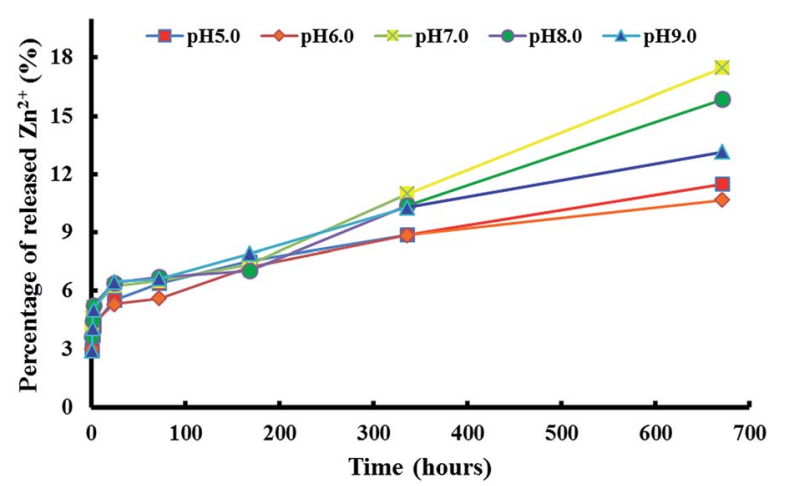

Fig. 8 Zinc ion released from a zein thin film incorporating $\mathrm{ZnO}$ crystals in an aqueous solution with different $\mathrm{pH}$ values.
It can be seen that the thin films kept a high stability under different $\mathrm{pH}$ conditions ranging from 5.0 to 9.0 and the highest percentage of released $\mathrm{Zn}^{2+}$ from the thin films was only $17.4 \%$ in 4 weeks. It has been well documented that $\mathrm{ZnO}$ can release $\mathrm{Zn}^{2+}$ and the excess zinc can penetrate through the biomembrane, affect the antioxidant defence system and further induce toxicity. ${ }^{9,35,36}$ Therefore, this result can explain why the thin film still has high antibacterial activities after 4 weeks of immersion. In summary, it will be very interesting to further study the application of a zein thin film incorporating ZnO crystals in food packaging due to its better film-forming effect and excellent antibacterial activities.

\section{Conclusion}

In summary, this study prepared a zein thin film incorporating $\mathrm{ZnO}$ crystals by the solution-casting method. Spindle-like zein conjugated ZnO crystals were synthesized via a green and gentle route. The participation of zein in zein conjugated $\mathrm{ZnO}$ crystals was key for the homogeneous distribution of ZnO crystals in the final zein thin film. At the same time, the synthesized zein thin film incorporating $\mathrm{ZnO}$ crystals showed high and long-term antibacterial activities against both Gram-negative and Grampositive pathogens. Moreover, the bacteriostatic effect is due to the released zinc ion from the thin film. These data can serve as a guide for the application of a zein thin film incorporating ZnO crystals to food packaging material.

\section{Acknowledgements}

This study was financially supported by the National Science Foundation of China (20971039 and 31000774), Program for Science \& Technology Innovation Talents in Universities of Henan Province (HASTIT, 16HASTIT049), Innovation Scientists and Technicians Troop Construction Projects of Henan Province (C20150018), Henan Science and Technology Research Program (162102210257), and Zhengzhou Science and Technology Plan Project (20150484).

\section{Notes and references}

1 N. B. Johnson, L. D. Hayes, K. Brown, E. C. Hoo and K. A. Ethier, MMWR Surveillance Summaries: Morbidity and Mortality Weekly Report, 2014, vol. 63, suppl. 4, p. 3.

2 H. Rostamzad, S. Y. Paighambari, B. Shabanpour, S. M. Ojagh and S. M. Mousavi, Food Packaging and Shelf Life, 2016, vol. 7, p. 1.

3 N. N. V. Long, C. Joly and P. Dantigny, Int. J. Food Microbiol., 2016, 220, 73.

4 C. Pires, C. Ramos, G. Teixeira, I. Batista, R. Mendes, L. Nunes and A. Marques, J. Food Eng., 2011, 105, 422.

5 P. Tongnuanchan, S. Benjakul, T. Prodpran and P. Songtipy, Int. J. Biol. Macromol., 2011, 48, 758.

$6 \mathrm{P}$. Appendini and J. H. Hotchkiss, Innovative Food Sci. Emerging Technol., 2002, 3, 113.

7 L. Wang, F. Liu, Y. Jiang, Z. Chai, P. Li, Y. Cheng, H. Jing and X. Leng, J. Agric. Food Chem., 2011, 59, 12411. 
8 A. Llorens, E. Lloret, P. Picouet and A. Fernandez, Int. J. Food Microbiol., 2012, 158, 113.

9 D. Salarbashi, S. A. Mortazavi, M. S. Noghabi, B. S. F. Bazzaz, N. Sedaghat, M. Ramezani and I. Shahabi-Ghahfarrokhi, Carbohydr. Polym., 2016, 140, 220.

10 J. Dong, Q. S. Sun and J. Y. Wang, Biomaterials, 2004, 25, 4691.

11 H. J. Wang, Z. X. Lin, X. M. Liu, S. Y. Sheng and J. Y. Wang, J. Controlled Release, 2005, 105, 120.

12 H. J. Wang, S. J. Gong, Z. X. Lin, J. X. Fu, S. T. Xue, J. C. Huang and J. Y. Wang, Biomaterials, 2007, 28, 3952.

13 Z. H. Qu, H. J. Wang, T. T. Tang, X. L. Zhang, J. Y. Wang and K. R. Dai, Acta Biomater., 2008, 4, 1360.

14 X. M. Liu, Q. S. Sun, H. J. Wang, L. Zhang and J. Y. Wang, Biomaterials, 2005, 26, 109.

15 S. J. Gong, H. J. Wang, Q. S. Sun, S. T. Xue and J. Y. Wang, Biomaterials, 2006, 27, 3793.

16 P. Argos, K. Pedersen, M. D. Marks and B. A. Larkin, J. Biol. Chem., 1982, 257, 9984.

17 A. S. Tatham, J. M. Field, V. J. Morris, K. J. I'Anson, L. Cardle, M. J. Dufton and P. R. Shewry, J. Biol. Chem., 1993, 268, 26253.

18 M. Abdollahi, M. Rezaei and G. Farzi, J. Food Eng., 2012, 111, 343.

19 D. S. Cha and M. S. Chinnan, Crit. Rev. Food Sci. Nutr., 2004, 44(4), 223.

20 B. Liu and H. C. Zeng, Langmuir, 2004, 20, 4196.

21 A. Vyalikh, P. Simon, E. Rosseeva, J. Buder, U. Scheler and R. Kniep, Sci. Rep., 2015, 5, 15797.
22 F. C. Meldrum, Int. Mater. Rev., 2003, 48(3), 187.

23 W. Z. Ostwald, J. Phys. Chem., 1900, 34, 495.

24 M. M. H. Farooqi and R. K. Srivastava, J. Alloys Compd., 2017, 691, 275.

25 R. Kripal, A. K. Gupta, R. K. Srivastava and S. K. Mishra, Spectrochim. Acta, Part A, 2011, 79, 1605.

26 K. Raja, P. S. Ramesh and D. Geetha, Spectrochim. Acta, Part A, 2014, 131, 183.

27 H. J. Wang, X. J. Yu, Y. Cao, B. Zhou and C. F. Wang, J. Inorg. Biochem., 2012, 113, 40.

28 Y. N. Chang, M. Zhang, L. Xia, J. Zhang and G. Xing, Materials, 2012, 5(12), 2850.

29 L. C. Ann, S. Mahmud, S. K. M. Bakhori, A. Sirelkhatim, D. Mohamad, H. Hasan, A. Seeni and R. A. Rahman, Ceram. Int., 2014, 40(2), 2993.

30 Y. W. Baek and Y. J. An, Sci. Total Environ., 2011, 409(8), 1603.

31 S. Sultana, M. Z. Rafiuddin, M. Z. Khan and M. Shahadat, J. Environ. Chem. Eng., 2015, 3(2), 886.

32 H. J. Wang, J. C. Huang, L. Hou, T. Miyazawa and J. Y. Wang, J. Mater. Sci.: Mater. Med., 2016, 27, 92.

33 L. Gutiérrez, A. Escudero, R. Battle and C. Nerín, J. Agric. Food Chem., 2009, 57, 8564.

34 L. Gutierrez, R. Battle, S. Andujar, C. Sanchez and C. Nerin, Packag. Technol. Sci., 2011, 24, 485.

35 U. I. Walther, S. C. Walther and O. Temruck, Toxicol. In Vitro, 2007, 21, 380.

36 B. Wihelm, U. I. Walther and B. Fichtl, Arch. Toxicol., 2001, $75,388$. 